\title{
NONLINEAR DIFFERENTIAL EQUATIONS
}

\author{
EDMUND PINNEY
}

1. Introduction. A few nonlinear differential equations have known exact solutions, but many which are important in applications do not. Sometimes these equations may be linearized by an expansion process in which nonlinear terms are discarded. When nonlinear terms make vital contributions to the solution this cannot be done, but sometimes it is enough to retain a few "small" ones. Then a perturbation theory may be used to obtain the solution. A differential equation may sometimes be approximated by an equation with "small" nonlinearities in more than one way, giving rise to different solutions valid over different ranges of its parameters.

There are two types of small nonlinearity problems. In the first type the nonlinearities occur in the most highly differentiated terms. These are very important in several physical theories. Carrier refers to them as "boundary layer problems" $[1 ; 2]$ in recognition of the application in which they had their first important development. They include many important nonlinear partial differential equations problems, as well as some ordinary nonlinear differential equations in which such phenomena as relaxation oscillations occur. Boundary layer problems are usually closely tied in with applications. Their theories have not yet received very general or exhaustive development, and much art and ingenuity has been called for in the work that has been done $([1]-[3])$.

The second type of nonlinearity problem is that in which nonlinearities do not occur in the most highly differentiated terms. In this case the theory has been developed farther, and something more nearly resembling a general method of attack is possible. Actually several such methods have been developed. Each has its own special merits and limitations. I will discuss one such method. This particular method has the advantage of wide scope and practicality of application, but is limited to a class of differential equations which is associated with nonconservative physical systems.

This method offers nothing new in the case of ordinary nonlinear differential equations of the second order, but has a practical advantage in the case of systems of equations (or, what comes to the same

An address delivered before the Los Angeles meeting of the Society on November 27, 1954, by invitation of the Committee to Select Hour Speakers for Far Western Sectional Meetings; received by the editors December 27, 1954. 
thing, vector differential equations of higher than the second order), certain functional equations, such as difference-differential equations, and some partial differential equations. Strictly formal solutions may be obtained by a number of devices analogous to the methods of van der Pol, Poincaré, Kryloff and Bogoliuboff, etc., with fewer manipulations than required by the present method, which is rigorous. This is all right when the necessary existence theorems are well known and fairly easily usable as in the case of second order ordinary differential equations. However in the more complicated cases mentioned, such existence theorems are frequently unknown, and, if known, are usually confined to the case of periodic solutions (ruling out the possibility of two oscillations having incommensurate periods) and are usually very difficult to apply. Thus they typically require calculations equivalent to obtaining the characteristic exponents arising in the application of Floquet theory to systems of linear equations with periodic coefficients. This is a formidable problem even when the original equation is a second order ordinary differential equation, but in that case the theory (Hill theory) is already known.

The present theory gets around this difficulty by basing the proof of the existence of a solution upon the "trend" functions that one would have to calculate anyway in order to obtain an approximation to the solution. Of course this process does not always work. As a general rule it does work in the case of equations arising in nonconservative physical systems. In this method an approximate solution to the nonlinear equation is developed, based on the linear system in which nonlinear terms are neglected. Such a theory cannot in itself settle the question of unboundedness of the solution, for, as the dependent variable increases, the nonlinear terms must ultimately dominate, thus invalidating the base of approximation. Therefore a prediction that the solution tends to infinity merely means that the theory fails. Indeed, the theory may fail for a prediction of large finite values of the solution when certain conditions fail to be satisfied. This will be called large solution failure.

The method is applied to van der Pol's equation in \$2, and carried through in detail. At the end of the section its principal features are recapitulated. About half of the calculation is devoted to proving the existence of the solution. In $\$ 3$ the method is applied to the much more elaborate problem of systems of nonlinear equations. Results only are given. In $\$ 4$ it is applied to a still more elaborate analysis of a partial differential equation occurring in a transmission line problem. For brevity much of the complete analysis is omitted, but enough is given to illustrate the ideas as well as some of the special 
problems and complexities that may be expected in nonlinear partial differential equations.

This work was sponsored by the Office of Naval Research at Stanford University and the University of California.

2. van der Pol's equation. van der Pol's equation is

$$
\frac{d^{2} y}{d t^{2}}+\epsilon\left(y^{2}-1\right) \frac{d y}{d t}+y=0,
$$

for $\epsilon$ small and positive. This may be written

$$
y^{\prime \prime}(t)+y(t)=f(t)
$$

where

$$
f(t)=\epsilon\left[1-y^{2}(t)\right] y^{\prime}(t) .
$$

Equation (2.2) has a particular solution

$$
\int_{0}^{t} \sin (t-\tau) f(\tau) d \tau
$$

so by (2.3),

$$
\begin{aligned}
& y(t)=y(0) \cos t+y^{\prime}(0) \sin t \\
& +\epsilon \int_{0}^{t} \sin (t-\tau)\left[1-y^{2}(\tau)\right] y^{\prime}(\tau) d \tau .
\end{aligned}
$$

This may be written

$$
y(t)=a_{+}(t) e^{i t}+a_{-}(t) e^{-i t},
$$

where

$$
a_{ \pm}(t)=\frac{1}{2} y(0) \pm \frac{1}{2 i} y^{\prime}(0) \pm \frac{\epsilon}{2 i} \int_{0}^{t} e^{\mp i r}\left[1-y^{2}(\tau)\right] y^{\prime}(\tau) d \tau
$$

Differentiating,

$$
a_{ \pm}^{\prime}(t)= \pm \frac{\epsilon}{2 i} e^{\mp i t}\left[1-y^{2}(t)\right] y^{\prime}(t)
$$

Then by (2.5),

$$
y^{\prime}(t)=i a_{+}(t) e^{i t}-i a_{-}(t) e^{-i t} .
$$

So far this development is exact, but it provides no method to compute the quantities $a_{ \pm}(t)$. Our main problem is to obtain approximate expressions for these quantities. As noted in the introduction, we 
shall use an approximate procedure which is valid only if the solution is bounded.

Let us provisionally assume that for some quantity $K$, independent of $t$,

$$
\left|a_{ \pm}(t)\right|<K
$$

for all $t \geqq 0$. Then we will show below that $a_{ \pm}(t)$ may be approximated by known functions $\rho_{ \pm}(t)$, the error bound $E=E(\epsilon, K)$ being expressed in terms of $\epsilon$ and $K$ :

$$
\left|a_{ \pm}(t)-\rho_{ \pm}(t)\right|<E(\epsilon, K) .
$$

Now let us not assume that (2.9) holds for all $t \geqq 0$, but that $\left|a_{ \pm}(0)\right|<K$. It will appear that the $\rho_{ \pm}(t)$ functions contain arbitrary constants which may be set so that $\rho_{ \pm}(0)=a_{ \pm}(0)$. Then the $\rho_{ \pm}(t)$ are completely known and an upper bound $K^{\prime}$ may be calculated:

$$
\left|\rho_{ \pm}(t)\right| \leqq K^{\prime}
$$

for all $t \geqq 0$. Suppose $K$ may be selected so that for $\epsilon$ in some neighborhood of $\epsilon=0$,

$$
K^{\prime}<K, \quad E(\epsilon, K)<K-K^{\prime} .
$$

Then by (2.10) and the triangle inequality it follows that (2.9) holds for all $t \geqq 0$ for $\epsilon$ in this neighborhood. It appears then that the boundedness of the $a_{ \pm}(t)$ is intimately associated with that of $\rho_{ \pm}(t)$. So long as we are able to select a $K$ greater than $K^{\prime}$ (which may depend on $\epsilon$ ) for which (2.11) holds, the validity of (2.9) follows for all $t \geqq 0$. When this cannot be done, the theory suffers large solution failure.

It should be emphasized that $K$ is not necessarily a least upper bound to the quantities $\left|a_{ \pm}(t)\right|$. Indeed, the present theory will not enable us to calculate this bound. $K$ may depend on $\epsilon$. Without loss in generality we can, and will, for convenience, assume that $K$ does not tend to 0 as $\epsilon \rightarrow 0$, i.e., $1 / K=O(1)$. Without specifying $K$ further we make the assumption (2.9), and proceed to calculate the function $E(\epsilon, K)$. With this and $K^{\prime}$, obtained from $\rho_{ \pm}(t)$, we then seek a $K$ greater than $K^{\prime}$ satisfying (2.11). Usually there are infinitely many possible values of $K$. The best is that which renders $E(\epsilon, K)$ smallest.

Now, assuming (2.9), by (2.5), (2.8),

$$
|y(t)|<2 K, \quad\left|y^{\prime}(t)\right|<2 K .
$$

Therefore by (2.7), assuming (as we shall hereafter) that $K \geqq 1$,

$$
\left|a_{ \pm}^{\prime}(t)\right|<4 \epsilon K^{3} .
$$


By (2.5), (2.7), (2.8),

$$
a_{ \pm}^{\prime}(t)=\frac{1}{2} \epsilon\left[\left(1-a_{+} a_{-}\right)\left(a_{ \pm}-a_{\mp} e^{\mp 2 i t}\right)-a_{ \pm}^{3} e^{ \pm 2 i t}+a_{\mp}^{3} e^{\mp 4 i t}\right]
$$

Now define

$$
\begin{aligned}
\bar{a}_{ \pm}(t)= & a_{ \pm}(t) \mp \frac{\epsilon}{4 i}\left(1-a_{+} a_{-}\right) a_{\mp} e^{\mp 2 i t} \pm \frac{\epsilon}{4 i} a_{ \pm}^{3} e^{ \pm 2 i t} \\
& \pm \frac{\epsilon}{8 i} a_{\mp}^{3} e^{\mp 4 i t} .
\end{aligned}
$$

Therefore by (2.9),

$$
\left|\bar{a}_{ \pm}(t)-a_{ \pm}(t)\right|<\frac{7}{8} \epsilon K^{3} .
$$

Differentiating (2.14) and inserting (2.12) and (2.13),

$$
\left|\bar{a}_{ \pm}^{\prime}(t)-\frac{1}{2} \epsilon\left(1-a_{+} a_{-}\right) a_{ \pm}\right|<\frac{17}{2} \epsilon^{2} K^{5} .
$$

Then by (2.15),

$$
\bar{a}_{ \pm}^{\prime}(t)=\frac{1}{2} \epsilon\left(1-\bar{a}_{+} \bar{a}_{-}\right) \bar{a}_{ \pm}+R_{ \pm}
$$

where

$$
\left|R_{ \pm}\right|<\frac{41}{4} \epsilon^{2} K^{5}
$$

We are interested in the degree to which the functions $\bar{a}_{ \pm}(t)$ may be approximated by the solutions $\rho_{ \pm}(t)$ of the equations

$$
\rho_{ \pm}^{\prime}(t)=\frac{1}{2} \epsilon\left(1-\rho_{+} \rho_{-}\right) \rho_{ \pm},
$$

which are the same as those in (2.16) except for the $R_{ \pm}$terms. Their solution is

$$
\rho_{ \pm}(t)=r(t) e^{ \pm i \phi}, \quad r(t)=\left(1+C e^{-\epsilon t}\right)^{-1 / 2},
$$

where $C$ and $\phi$ are real constants.

The "trend functions" $\rho_{ \pm}(t)$ tend to $e^{ \pm i \phi}$ as $t \rightarrow \infty$. What about the quantities $\bar{a}_{ \pm}(t)$ ? To answer this, construct an $r, \theta, t$-cylindrical coordinate system, and plot the surfaces $r=r(t)$, which are surfaces of revolution about the $t$-axis. This will give a one-parameter family of surfaces filling the space. These surfaces will be called "trend surfaces." As $t \rightarrow \infty$, each trend surface approaches the cylinder $r=1$ asymptotically. The space curves $r=r(t), \theta=\phi$ will be called "trend lines." Now write 


$$
\bar{a}_{ \pm}(t)=A(t) e^{ \pm i \theta(t)}
$$

where $A(t)$ and $\theta(t)$ are real. Then by (2.16),

$$
A^{\prime}(t)=\frac{1}{2} \epsilon\left(1-A^{2}\right) A+R, \quad \theta^{\prime}(t)=I / A,
$$

where

$$
R \pm i I=R_{ \pm} e^{\mp i \theta} .
$$

The space curve $r=A(t), \theta=\theta(t)$ will at every point cut a local trend line from which its direction angles will differ by $R / A$ and $I / A$.

Specifically, write $\bar{a}_{ \pm}(0)=A_{0} e^{ \pm i \phi_{0}}$, and let $\rho_{ \pm}(t)$ denote the trend function whose trend line passes through $\left(A_{0}, \phi_{0}, 0\right)$, i.e., $\rho_{ \pm}(0)=\bar{a}_{ \pm}(0)$, and $r(0)=A_{0}, \phi=\phi_{0}$. How far away from $\rho_{ \pm}(t)$ can $\bar{a}_{ \pm}(t)$ get for $t>0$ ? In other words, how far can $A(t)$ get from $r(t)$, and $\theta(t)$ from $\phi_{0}$ ? First, suppose $A(t)>r(t)$. Then $A(t)-r(t)$ grows only if $A^{\prime}(t)$ $-r^{\prime}(t)>0$. By (2.19), (2.21), this implies

$$
\frac{1}{2} \epsilon\left[\left(1-A^{2}\right) A-\left(1-r^{2}\right) r\right]+R>0 .
$$

Therefore

$$
(A-r)\left(A^{2}+A r+r^{2}-1\right)<2 R / \epsilon .
$$

Therefore when $A_{0}>1 / 3$,

$$
A-r<2 R /\left[\epsilon\left(3 A_{0}^{2}-1\right)\right],
$$

so by (2.17), (2.22),

$$
A(t)-r(t)<(99 / 4) \epsilon K^{5} /\left(3 A_{0}^{2}-1\right) .
$$

When $A_{0}<1 / 3$ the analysis is slightly more complicated because the trend lines themselves are at first diverging, but we again get $A(t)-r(t)=O\left(\epsilon K^{5}\right)$. The same order estimate is obtained when $A(t)<r(t)$. By (2.17), (2.21), (2.22), $\theta(t)=\phi+t O\left(\epsilon^{2} K^{5}\right)$,

$$
\bar{a}_{ \pm}(t)=r(t) \exp \left( \pm i\left[\phi+Ю\left(\epsilon^{2} K^{5}\right)\right]\right)+O\left(\epsilon K^{5}\right) .
$$

From (2.15) we have $a_{ \pm}(t)=\rho_{ \pm}(t) \exp \left( \pm i t O\left(\epsilon^{2} K^{5}\right)\right)+O\left(\epsilon K^{5}\right)$. In view of (2.19), $\left|\rho_{ \pm}(t)\right|$ has an upper bound $K^{\prime}=\max \left(1, A_{0}\right)$. Since $A_{0}=O(1)$ we can take $K^{\prime}=O(1)$. Then (2.11) is satisfied if $K-K^{\prime}$ $>O\left(\epsilon K^{5}\right)$. This is clearly possible with $K=O(1)$, so that $a_{ \pm}(t)=\rho_{ \pm}(t)$ - $\exp \left( \pm i t O\left(\epsilon^{2}\right)\right)+O(\epsilon)$. By (2.5), (2.8), (2.19), 


$$
\begin{aligned}
y(t) & =2 r(t) \cos \left[\left\{1+O\left(\epsilon^{2}\right)\right\} t+\phi\right]+O(\epsilon), \\
y^{\prime}(t) & =-2 r(t) \sin \left[\left\{1+O\left(\epsilon^{2}\right)\right\} t+\phi\right]+O(\epsilon) .
\end{aligned}
$$

The principal features of this method may now be pointed out. First the equation is written in the form (2.2) in which the principal linear part is on the left while the term on the right contains the nonlinear terms. This equation is "solved" in the same manner as the linear nonhomogeneous equation is solved, getting an integral equation (2.4). We write this in a form (2.5) resembling the solution to the linear homogeneous equation $(f=0$ in (2.2)) except that the quantities $a_{ \pm}(t)$ are not now constant. However they have the property that when differentiated, giving (2.7), the integral sign is removed. Substituting in the expressions for $y$ and $y^{\prime}$ in terms of $a_{ \pm}$, the first order differential equations for $a_{ \pm}(t)$ are given in (2.13). These equations are more complicated than the original equation (2.1), but they provide the basis for making an approximation. The intuitive reason to suspect this virtue in (2.13) is as follows. Since the equation has only a small nonlinearity when $\epsilon$ is small, its solution will be almost like a linear solution. Therefore the quantities $a_{ \pm}(t)$, although not actually constant, should vary only slowly with $t$. Therefore on the right-hand side of (2.13) the terms involving $e^{\mp 2 i t}$, $e^{ \pm 2 i t}$, and $e^{\mp 4 i t}$ oscillate in almost the same way as these quantities do themselves and so tend to cancel themselves out over a long $t$ interval in comparison with the other, nonoscillatory, terms. The introduction of the quantities $\bar{a}_{ \pm}(t)$ and $\rho_{ \pm}(t)$ leads to simplified equations in which the oscillatory terms are absent. The $\rho_{ \pm}(t)$ quantities are approximations to the $a_{ \pm}(t)$ quantities because of their exponential approach to their asymptotic limits. An approximate solution to the differential equation may then be written in terms of them. The accuracy of this approximation involves the analysis of the $K$ and $K^{\prime}$ quantities and $E(\epsilon, K)$ as indicated in (2.9)-(2.11).

These same ideas may be applied to other and more complicated nonlinear situations. In fact, it is in the more complicated problems that this method has an advantage of practicality which it does not have in this problem.

3. Systems of nonlinear differential equations. The foregoing procedure may be applied to systems of equations. For brevity, results only will be given here. We shall consider a system

$$
\frac{d y_{i}}{d t}=\sum_{\alpha=1}^{m} A_{i \alpha} y_{\alpha}+\epsilon f_{i}(y, t), \quad i=1,2, \cdots, m,
$$


the quantities in which are defined as follows:

(i) the $A_{i \alpha}$ are constants and $\epsilon$ is a small parameter;

(ii) $y=y(t)$ is the matrix $\left(y_{1}(t), y_{2}(t), \cdots, y_{m}(t)\right)$;

(iii) $f_{i}(y, t)$ is a polynomial of degree $n$ in the elements of $y$; the terms of degree $n$ have coefficients that are all $O(1)$ in $\epsilon$ as $\epsilon \rightarrow 0$;

(iv) $f_{i}(y, t)$ depends on $t$ explicitly only as a polynomial in circular functions of $t$;

(v) for $b_{1}, b_{2}, \cdots, b_{m}$ arbitrarily preassigned quantities,

$$
y_{i}(0)=b_{i}, \quad i=1,2, \cdots, m .
$$

It is an elementary matter to express these equations and conditions more completely in matrix notation, omitting the subscripts and summation sign from (3.1), say. However many of our manipulations lie outside the limited class that can be done with this abbreviated notation, so the present more descriptive but slightly longer hybrid notation seems more efficient.

To discuss the system (3.1), let $S$ denote the set of characteristic roots $z_{j}$ (of multiplicity $\mu_{j}$ ) of the characteristic equation

$$
D(z) \equiv\left|A_{i j}-z \delta_{i j}\right|=0,
$$

and let $D_{j i}(z)$ be the cofactor of $A_{i j}-z \delta_{i j}$ in the determinant of (3.3). Let $\lambda_{1}, \lambda_{2}, \cdots, \lambda_{m}$ be an arbitrarily chosen set of constants not all of which are zero, and let $\nu_{1}, \nu_{2}, \cdots, \nu_{m}$ be another such set. Define the quantities

$$
\begin{aligned}
D_{r}(z) & =\sum_{s=1}^{m} \lambda_{s} D_{r s}(z), \quad \bar{D}_{s}(z)=\sum_{r=1}^{m} \nu_{r} D_{r s}(z), \\
\bar{D}(z) & =\sum_{r=1}^{m} \nu_{r} D_{r}(z)=\sum_{s=1}^{m} \lambda_{s} \bar{D}_{s}(z)=\sum_{r=1}^{m} \sum_{s=1}^{m} \lambda_{s} \nu_{r} D_{r s}(z), \\
\bar{D}_{i \alpha}(z) & =\sum_{r=1}^{m} \sum_{s=1}^{m} \lambda_{s} \nu_{r} D_{i s ; r \alpha}(z),
\end{aligned}
$$

where $D_{i s ; r \alpha}(z)$ is the cofactor of $A_{\alpha r}-z \delta_{\alpha r}$ in the determinant $D_{i s}(z)$. Define

$$
\begin{aligned}
G_{i}(z) & =-D_{i}(z) / \bar{D}(z), \\
F(z, y, t) & =\sum_{\alpha=1}^{m} \bar{D}_{\alpha}(z) f_{\alpha}(y, t) .
\end{aligned}
$$

The quantities $y_{i}(t)$ may be expressed in terms of certain quantities $a_{j, q}(t)$ in a manner analogous to $(2.5)$ : 


$$
y_{i}(t)=\sum_{S^{j}} e^{z_{j} t} \sum_{q=0}^{\mu_{j}-1} \frac{\mu_{j} ! G_{i}^{(q)}\left(z_{j}\right)}{q ! D^{\left(\mu_{j}\right)}\left(z_{j}\right)} a_{j, \mu_{j-1-q}}(t),
$$

where the $a_{j, q}(t)$ quantities may be expressed in terms of integrals of $F(z, y, t)$ in a manner analogous to $(2.6)$, and satisfy differential equations analogous to (2.7).

Again, as in $\$ 2$, we are interested in bounded solutions and introduce a quantity $K$ of the same type as that of $\$ 2$, except that we have

$$
\left|e^{z_{j} t} a_{j q}(t)\right|<K \text { for } z_{j} \text { in } S, \quad q=0,1, \cdots, \mu_{j}-1,
$$

instead of (2.9). As in (2.11) we require that

$$
K>K^{\prime}, \quad E(\epsilon, K)<K-K^{\prime}
$$

as $\epsilon \rightarrow 0$ to avoid large solution failure.

The quantities $y_{i}(t)$ in (3.7) are substituted into (3.6) and simplified. We write

$$
F(z, y(t), t)=\sum_{S^{\prime}} e^{z_{j} t} g_{j}(z, a(t), t)+\sum_{S^{\prime}} e^{z_{j}^{\prime} t} G_{j}(z, a(t)),
$$

in which the following rules govern the indicated expansion:

(i) the terms included in the $S$ summation must be such that

$$
\frac{\partial}{\partial t} g_{j}(z, a, t)=e^{-z_{j} t} O\left(\epsilon K^{2 n-1}\right)
$$

(ii) the $S^{\prime}$ summation is over all terms in the expansion of the left-hand side of (3.10) not included in the $S$ summation. However, we shall impose the further restriction that

$$
1 /\left|z_{j}-z_{k}^{\prime}\right|=O(1)
$$

as $\epsilon \rightarrow 0$ for all $z_{j}$ in $S$ and all $z_{\boldsymbol{k}}^{\prime}$ in $S^{\prime}$.

Condition (i) simply defines the terms which are to be included in the $S$ summation. However condition (3.12) in (ii) implies a new restriction on $F$ and on the characteristic roots. A difficulty might seem to appear in the use of (3.11), because the quantity $K$ is not yet known. In practice this is generally not significant because (3.11) usually implies a restriction upon certain parameters in the equations (3.1) which need not be made precise till later.

In place of equation (2.18), we now get "trend equations,"

$$
\frac{d \rho_{j q}(t)}{d t}=\rho_{j, q-1}(t)+\epsilon g_{j}^{(q)}\left(z_{j}, \rho(t), t\right) / q !
$$


for $z_{j}$ in $S, q=0,1, \cdots, \mu_{j}-1$, and for $\rho_{i,-1}(t) \equiv 0$. Here $g_{j}^{(q)}(z, a, t)$ denotes $\left(\partial^{a} / \partial z^{a}\right) g_{j}(z, a, t)$.

When the equations in (3.13) are solved, the "trend functions" $\rho_{j q}(t)$ can be analysed in a way similar to that used to analyse the trend functions in $\$ 2 .\left|\rho_{j q}(t)\right|$ may be plotted against $t$ on a $j q$-trend modulus plane, and arg $\left[\rho_{j q}(t)\right]$ may be plotted against $t$ on a " $j q-$ trend argument cylinder," which is a cylindrical surface having $t$ as axial distance and $\arg \left[\rho_{j q}(t)\right]$ as azimuthal angle.

We shall designate as "principal trend functions" the set of trend functions $\rho_{j q}(t)$ which takes on some prescribed initial conditions. Since the solution of (3.13) involves constants of integration, this is only one set in a family. The behavior of others "near" this set is very important, as it was in $\$ 2$.

Theorem 1. On each jq-trend modulus plane let $\Delta\left|\rho_{j q}(t)\right|$ denote the difference in ordinate between the principal jq-trend modulus and neighboring trend moduli; on each jq-trend argument cylinder let $\Delta$ arg $\left[\rho_{j q}(t)\right]$ denote the difference in azimuthal angle between the principal jq-trend argument and neighboring trend arguments. Suppose

$$
\Delta\left|\rho_{j q}(t)\right|=f_{j q}(t) e^{-\left(x_{j}+\kappa\right) t}, \quad \Delta \arg \left[\rho_{j q}(t)\right]=g_{j q}(t) e^{-\kappa t},
$$

where $x_{j}=\operatorname{Re}\left(z_{j}\right), \kappa>0$, and

$$
f_{j q}^{\prime}(t) / f_{j q}(t) \leqq 0, \quad g_{j q}^{\prime}(t) / g_{j q}(t) \leqq 0 .
$$

Then for $t \geqq 0, i=1,2, \cdots, m$,

$$
\begin{aligned}
y_{i}(t)= & \sum_{S} \sum_{q=0}^{\mu_{j}-1} \frac{\mu_{j} ! G_{j}^{(q)}\left(z_{j}\right)}{q ! D^{\left(\mu_{j}\right)}\left(z_{j}\right)} e^{z_{j} t} \rho_{j, \mu_{j-1-q}}(t) \\
& +O\left(\epsilon K^{n}\right)+O\left(\epsilon^{2} K^{2 n-1} / \kappa\right) .
\end{aligned}
$$

Unless $\kappa>0$ and the conditions in (3.15) are satisfied, the theory fails. When the conditions in (3.15) are not satisfied, sometimes a simple special discussion will insure the success of the theory, as in $\$ 2$ in the case $A_{0}<1 / 3$.

Suppose a quantity $K^{\prime}$, independent of $t$, exists such that for $z_{j}$ in $S, q=0,1, \cdots, \mu_{j}-1$,

$$
\left|e^{z_{j} t} \boldsymbol{\rho}_{j q}(t)\right| \leqq K^{\prime},
$$

and that $K^{\prime}$, which may depend on $\epsilon$, satisfies

$$
\begin{aligned}
& K^{\prime}=o\left(\epsilon^{-1 /(n-1)}\right), \quad 1 / K^{\prime}=O(1), \\
& K^{\prime}=o\left(\kappa^{1 /(2 n-1)} / \epsilon^{2 /(2 n-1)}\right),
\end{aligned}
$$


as $\epsilon \rightarrow 0$. Then (3.9) is satisfied for a value of $K$ such that

$$
K=O\left(K^{\prime}\right)
$$

and the error estimate in (3.16), as well as the restriction implied in (3.11), may be made definite.

4. A partial differential equation application. A nonlinear transmission line. Our method may also be applied to partial differential equations problems which are not of the boundary layer type. The general features of the method are the same although there are some added complications of detail.

We shall consider resonance oscillations in a transmission line in which the series resistance, series inductance, and shunt capacitance are constant as usual, but in which the shunt conductance varies with transverse voltage. The mechanism by which this is accomplished is not our direct concern here, but there are such mechanisms, gaseous discharge tubes, for instance. The series resistance and shunt conductance will be assumed to be small.

We shall suppose the transmission line to have a finite length, and to be short circuited at the end. The case when the line is terminated by a nonzero impedance requires a slightly different treatment as will be noted below. The other end of the line will be driven by a harmonically oscillating generator of frequency $\alpha$ near one of the natural frequencies of the linearized system. Under these conditions the current flowing in the line and the voltage across the line may be expected to be rather large, i.e., a "resonant" condition prevails. If $V$ is the transverse voltage across the line, $x$ is the distance along the line, and $t$ is time,

$$
\begin{array}{lll}
V=V_{0} \cos (\alpha t) & \text { when } & x=0, \\
V=0 & \text { when } & x=l .
\end{array}
$$

In the range $0<x<l$, but not too near $x=0$ or $x=l$, $V$ may be expected to be large compared to $V_{0}$.

Let $L$ be the series inductance; $R$, the series resistance; $C$, the shunt capacitance; and $G$, the shunt conductance, all per unit length, and uniformly distributed. Let $I$ be the current in the line. $L, R$, and $C$ are constant, but $G$ is a function of $V$. Then, by $[4$, p. 550],

$$
\frac{\partial V}{\partial x}+L \frac{\partial I}{\partial t}+R I=0, \quad \frac{\partial I}{\partial x}+C \frac{\partial V}{\partial t}+G V=0 .
$$

It will be convenient to introduce dimensionless variables 


$$
X=\pi x / l, \quad T=\pi c t / l
$$

where

$$
c=1 /(L C)^{1 / 2} .
$$

Substituting these into (4.2) and eliminating $I$,

$$
V_{X X}-V_{T T}=F\left(\epsilon V, \epsilon V_{T}\right)=u(X, T),
$$

where $\epsilon$ is a conveniently chosen small quantity such that

$$
F\left(\epsilon V, \epsilon V_{T}\right)=\left(\pi^{2} / l^{2}\right)\left\{\left[L \frac{d}{d V}(G V)+R C\right](\pi c / l) V_{T}+R G V\right\} .
$$

$F$ will be assumed to be a polynomial of degree $N$ in its arguments.

Our problem is to solve equation (4.5) subject to the boundary conditions (4.1). Although the main ideas are the same as in $\$ 2$, the manipulative machinery is substantially heavier, and for brevity much of it must be suppressed in this paper.

For $m$ an integer define $\delta$ by

$$
\alpha=m(1+\delta) \pi c / l .
$$

Then $|\delta| \ll 1$ implies resonance. The boundary conditions (4.1) may be written

$$
\begin{array}{lll}
V=V_{0} \cos m(1+\delta) T & \text { when } & X=0, \\
V=0 & \text { when } & X=\pi .
\end{array}
$$

Then a solution to (4.5), satisfying (4.8) is

$$
\begin{gathered}
V=V(X, T)=(1-X / \pi) V_{0} \cos m(1+\delta) T \\
-\frac{1}{2} \int_{0}^{T} \int_{X-T+\tau}^{X+T-\tau} U(\xi, \tau) d \xi d \tau
\end{gathered}
$$

where

(4.10) $U(X, T)=u(X, T)-m^{2}(1+\delta)^{2}(1-X / \pi) V_{0} \cos m(1+\delta) T$. (4.9) is analogous to (2.4).

At this point it may be worth interpolating that had our transmission line been terminated by a nonzero impedance $Z$ rather than short-circuited, instead of (4.9) we would have taken

$$
\begin{aligned}
V \equiv V(X, T)=V_{0} e^{-X l R /(\pi Z)} \cos m(1+\delta)(T-X c L / Z) \\
-\frac{1}{2} \int_{0}^{T} \int_{X-T+\tau}^{X+T-\tau} U(\xi, \tau) d \xi d \tau .
\end{aligned}
$$


The subsequent treatment of equation (4.11) would be closely analogous to that of equation (4.9).

To resume, (4.9) will satisfy the boundary conditions (4.8) provided the range of definition of the function $U(X, T)$ is extended beyond $0<X<\pi$ in such a way that it is odd in $X$ and periodic of period $2 \pi$ in $X$. It can be shown to be of bounded variation in $X$ and therefore expandable in a Fourier sine series:

$$
U(X, T)=i \sum_{n=-\infty}^{\infty} U_{n}(T) e^{i n X},
$$

where

$$
U_{0}(T)=0,
$$

$$
\begin{aligned}
U_{n}(T)= & -(1 / \pi) \int_{0}^{\pi} F\left(\epsilon V(\xi, T), \epsilon V_{T}(\xi, T)\right) \sin (n \xi) d \xi \\
& +\left(m^{2} / n \pi\right)(1+\delta)^{2} V_{0} \cos m(1+\delta) T
\end{aligned}
$$

for $n \neq 0$.

Define

$$
a_{n}(s)=\int_{0}^{s} U_{n}(\tau) e^{-i n \tau} d \tau
$$

so that

$$
a_{n}^{\prime}(s)=U_{n}(s) e^{-i n s},
$$

and define

$$
\begin{aligned}
W(X, a(s))= & \frac{1}{2} \int_{0}^{s}\left\{\int_{0}^{X-\tau} U(\xi, \tau) d \xi\right. \\
& \left.-\int_{0}^{\pi} U(\xi, \tau)(1-\xi / \pi) d \xi\right\} d \tau \\
= & \sum_{n=-\infty}^{\infty}(1 / 2 n) a_{n}(s) e^{i x X} .
\end{aligned}
$$

Then it may be shown that

$$
\begin{aligned}
V(X, T)= & (1-X / \pi) V_{0} \cos m(1+\delta) T \\
& +W(T-X, a(T))-W(T+X, a(T)) .
\end{aligned}
$$

Returning to the problem in $\$ 2$, equations (4.16) and (4.17) are 
analogous to (2.5). (4.14) is analogous to (2.6), and (4.15) is analogous to (2.7).

Again, as in $\S \S 2$ and 3, we are interested in bounded solutions, and introduce a similar quantity $K$, except that in place of (2.9) we have, for $n \neq 0$,

$$
\left|a_{n}(t)\right|<K /|n| .
$$

As in (2.11), if large solution failure is to be avoided,

$$
K>K^{\prime}, \quad E(\epsilon, K)<K^{\prime}-K .
$$

In place of (2.18), we now get "trend equations,"

$$
\rho_{n}^{\prime}(T)=g_{n, n}(\rho(T), \delta T)
$$

for $n \neq 0$, where

$$
g_{n, k}(a(s), \sigma)=(1 / 2 \pi) \int_{-\pi}^{\pi} U_{n}(a(s), \sigma, \tau) e^{-i k \tau} d \tau,
$$

where

$$
\begin{aligned}
& U_{n}(a(s), \sigma, T)=\left(m^{2} / n \pi\right)(1+\delta)^{2} V_{0} \cos m(T+\sigma) \\
&-(1 / \pi) \int_{0}^{\pi} F(\epsilon[W(T-\xi, a(s))-W(T+\xi, a(s))], \\
&\left.\epsilon\left[W_{1}(T-\xi, a(s))-W_{1}(T+\xi, a(s))\right]\right) \sin (n \xi) d \xi .
\end{aligned}
$$

The conditions that the "trend functions" $\rho_{n}(T)$ must satisfy in order to approximate the functions $a_{n}(T)$ are precisely those given in (3.14) and (3.15) with $x_{j}=0$ and with the double $j q$ subscripts replaced by $n$. If one solves (4.20) and verifies these conditions, then the solution is obtained by substituting

$$
\begin{aligned}
W(X, a(T))= & W(X, \rho(T))+O(\delta / \kappa)+O(1) \\
& +O\left(\epsilon^{N} K^{N}\right)+(\epsilon / \kappa)\left[O(1)+O\left(\epsilon^{2 N-1} K^{2 N-1}\right)\right] .
\end{aligned}
$$

Now suppose a quantity $K^{\prime}$, independent of $t$, exists such that for $n \neq 0$,

$$
\left|\rho_{n}(T)\right| \leqq K^{\prime} /|n|,
$$

and that $K^{\prime}$, which may depend on $\epsilon$, satisfies

$$
\begin{aligned}
K^{\prime} & =o\left(\epsilon^{-N /(N-1)}\right), & 1 / K^{\prime} & =O(1), \\
\epsilon / \kappa & =o\left(K^{\prime}\right), & K^{\prime} & =o\left(\kappa^{1 /[2(N-1)]} \epsilon^{-N /(N-1)}\right),
\end{aligned}
$$

as $\epsilon \rightarrow 0$. Then (4.19) is satisfied if 


$$
K=O\left(K^{\prime}\right)
$$

and the error estimate in (4.23) may be made definite.

Since (4.20) represents an infinite system of differential equations, its solution may not be simple. One method of attack is to study a generating function

$$
R(X, T)=\epsilon W(X-\delta T, \rho(T))=\epsilon \sum_{n=-\infty}^{\infty}(1 / 2 \pi) \rho_{n}(T) e^{i n(X-\delta T)} .
$$

This may be shown to satisfy the equation

$$
R_{X T}(X, T)+\delta R_{X X}(X, T)
$$

$$
\begin{aligned}
& +(\epsilon / 8 \pi) \int_{-\pi}^{\pi}\left\{F\left(R(X, T)-R(\xi, T), R_{X}(X, T)-R_{\xi}(\xi, T)\right)\right. \\
& \left.\quad-F\left(R(\xi, T)-R(X, T), R_{\xi}(\xi, T)-R_{X}(X, T)\right)\right\} d \xi \\
& +\epsilon(m / 2 \pi)(1+\delta)^{2} V_{0} \sin (m X)=0 .
\end{aligned}
$$

Since $F$ is a polynomial the integrand can be expanded. The integral is seen to be a polynomial in $R(X, T)$ and $R_{X}(X, T)$ whose coefficients are averages of polynomials of these two quantities taken over the range $-\pi<X<\pi$.

By (4.27), $R(X, T)$ must be periodic of period $2 \pi$ in $X$. It is interesting to note that only odd terms in the expansion of $F$ contribute to the integral in (4.28).

It may appear that we are replacing the partial differential equation (4.5) by a worse equation in (4.28). However (4.28) has some real advantages. In a problem of this type we are mainly interested in the "steady state" behavior of the system for $t$ large. The physical nature of the problem strongly suggests that the quantities $\rho_{n}(T) e^{-i n \delta T}$ approach constants asymptotically as $T \rightarrow \infty$, so that, by (4.27), the steady state behavior of the system is determined by $R(X, \infty)=R(X)$, say. By (4.28), $R(X)$ satisfies an ordinary differential equation of the second order whose coefficients contain mean values of polynomials in $R(X)$ and $R^{\prime}(X)$. If the small nonlinear part of the shunt conductance is comparable in magnitude to the small linear part, then the equation for $R(X)$ is strongly nonlinear and its numerical computation is necessary. In this case the transmission line may be rather strongly excited, not only in the mode nearest the exciting frequency, but in all others as well. On the other hand, when the nonlinear part of the shunt conductance is small compared to the linear part, the equation for $R(X)$ is weakly nonlinear, and may be solved analytically by the usual perturbation 
methods. In this case, only the mode nearest the exciting frequency is excited strongly.

The validity of the solution corresponding to $R(X)$ can be established if conditions (3.14) and (3.15) are satisfied. To verify these we must know the asymptotic behavior of $\rho_{n}(T)$ as $T \rightarrow \infty$. This can be found from a perturbation analysis of the $R(X)$ solution. That is, we substitute

$$
R(X, T)=R(X)+\eta(X, T)
$$

into (4.28) and discard terms of higher powers than the first in $\eta$. This may turn out to be quite a difficult analysis comparable to, or worse than the analysis of the Hill equation.

Since our object is to illustrate a general method and to give no more than a glimpse of the special problems that are peculiar to special applications of this method, it does not seem appropriate to go further into the analysis of the function $R(X, T)$, since this is a matter of importance only to those who may be interested in this specific physical problem. This latter group may be satisfied with less than a complete analysis, for instance, with a determination of $R(X)$ only, the validity of the resulting solution being established by physical or experimental means.

\section{REFERENCES}

1. G. F. Carrier, Boundary layer problems in applied mechanics, "Advances in Applied Mechanics," vol. III, Academic Press, 1953.

2. - Boundary layer problems in applied mathematics, Communications on Pure and Applied Mathematics vol. 7 (1954) pp. 11-17.

3. M. J. Lighthill, A technique for rendering approximate solutions to physical problems uniformly valid, Philosophic Magazine vol. 7, XL (1949) p. 1179.

4. J. A. Stratton, Electromagnetic theory, McGraw-Hill, 1941.

University of California, Berkeley 